\title{
Trochlear Nerve Disorder
}

National Cancer Institute

\section{Source}

National Cancer Institute. Trochlear Nerve Disorder. NCI Thesaurus. Code C78395.

A non-neoplastic or neoplastic disorder affecting the trochlear nerve (IVth cranial nerve). 